\title{
Antibiotic stewardship program in the Capital Health Region of British Columbia
}

\author{
Richard L Bachand BSc (Pharm) PharmD
}

$\mathrm{T}$ he Capital Health Region of British Columbia was formed on April 1, 1998 and comprises several partners covering Greater Victoria, and from Port Renfrew to the Saannich Peninsula and the southern Gulf Islands. Partners are the former Greater Victoria Hospital Society (Victoria General Hospital, Royal Jubilee Hospital, Gorge Road Hospital and Fairfield Health Centre), the former Juan de Fuca Hospital Society (Aberdeen Hospital, Priory Hospital, Glengarry Hospital and Mt Tolmie Hospital), the Saanich Peninsula Hospital, the Queen Alexandra Centre for Children's Health, the Lady Minto Hospital (Salt Spring Island), Community and Public Health Services, and Mental Health Services.

Demographics of the Capital Health Region are astounding. There are approximately 840 acute care beds and 3100 extended/long term care beds. Employees number nearly 10,000, and physicians with prescribing privileges, most of whom are general practitioners, number approximately 850 .

The present report outlines strategies promoting optimal antimicrobial use that were undertaken in the acute care set- ting of the Victoria General and Royal Jubilee hospitals. While many of the principles were applied to acute care, they may also be applicable to extended care/long term care settings.

\section{ANTIMICROBIAL BUDGET}

Perhaps the most difficult challenge of any health organization is to maintain fiscal accountability. This is particularly true for pharmacy departments. Conventional budgeting practices for most hospitals include allocating the drug budget to pharmacy, such that pharmacy is responsible for keeping expenditures within the allocated budget allowances. Pressure over the past several years has been to decrease drug expenditures despite increasing patient acuity, decreasing hospitalization days and increasing hospital admissions. Program management proposals most often shift drug expenditures to the shared team responsibility of medical, nursing and pharmacy departments.

Antimicrobial drug expenditures for the Royal Jubilee

TABLE 1

Yearly antimicrobial expenditures (\$ million) for the Victoria General and Royal Jubilee hospitals in the Capital Health Region of British Columbia from 1992 to 1998

\begin{tabular}{|c|c|c|c|c|c|c|}
\hline & $1992 / 1993$ & 1993/1994 & 1994/1995 & $1995 / 1996$ & 1996/1997 & 1997/1998 \\
\hline Total drug costs & 7.18 & 7.54 & 7.73 & 7.47 & 8.20 & 7.87 \\
\hline Total antimicrobial costs & 1.61 & 1.43 & 1.38 & 1.31 & 1.27 & 1.09 \\
\hline $\begin{array}{l}\text { Antimicrobial cost percentage of } \\
\text { total of drug costs }\end{array}$ & 23 & 19 & 18 & 18 & 16 & 14 \\
\hline
\end{tabular}

Capital Health Region, Victoria, British Columbia

Correspondence and reprints: Dr RL Bachand, Royal Jubilee Hospital,1900 Fort Street, Victoria, British Columbia V8R 1 J8. Telephone 250-370-8443, fax 250-370-8615, e-mail rbachand@caphealth.org 


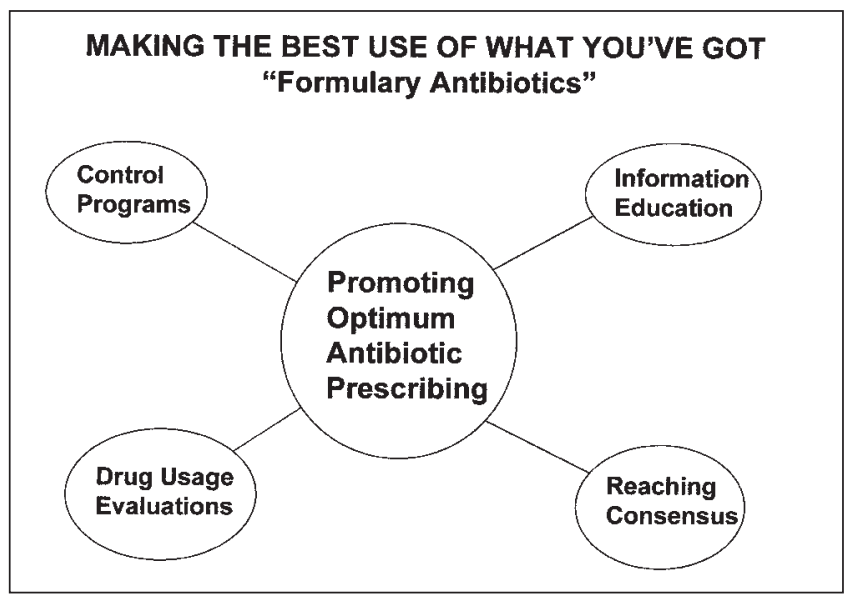

Figure 1) Strategies employed to promote optimal prescription of antibiotics

Hospital and Victoria General Hospital over the past five years (1992 to 1998) have steadily decreased, amounting to savings of $\$ 360,000$ (Table 1). The percentage of the total drug expenditures that antimicrobial agents accounted for from 1992 to 1998 has also decreased significantly from $23 \%$ to $16 \%$.

\section{STRATEGIES FOR PROMOTING OPTIMAL ANTIBIOTIC USE}

The Antimicrobial Review Subcommittee is one of the most valued and hardworking subcommittees of the Pharmacy and Therapeutics Committee. In the mid-1980s, the subcommittee was formed and, at present, comprises two infectious disease physicians, one medical microbiologist, one internal medicine physician and three clinical pharmacists. Representation by the department of surgery has been sporadic at best. Although surgeon members have been appointed, few have been able to attend the meetings. In view of the importance of their input, issues related to the depratment of surgery are taken by a member of the subcommittee to the most appropriate surgical group (eg, general surgery, orthopedic or cardiac) for review and ratification as required.

Antimicrobial agents are one of the most frequently prescribed types of drugs for the hospitalized patient. With few exceptions, physicians regularly prescribe antimicrobial drugs. The Antimicrobial Review Subcommittee has employed a number of strategies in the past in an effort to influence the prescribing of antimicrobial drugs (Figure 1). Some of the methods have been successful, while some have not.

The formulary: The hospital formulary continues to be an important first step in promoting appropriate drug use. Drugs cannot be abused if they are not stocked. All issues relating to antimicrobial drugs are referred by the Pharmacy and Therapeutics Committee to the Antimicrobial Review Subcommittee, which makes recommendations. It is extremely important that the policies considered by the committees can be carried out efficiently. For example, if use of an antimicrobial drug is restricted to a disease condition or a particular service, then processes should be in place to ensure that before dispensing medication, the pharmacy is aware that antimicrobial use con-
TABLE 2

Antimicrobial control programs employed in the acute care settings of the Victoria General and Royal Jubilee Hospitals in the capital health region of British Columbia

\begin{tabular}{ll}
\hline Control program & Examples \\
\hline Therapeutic & Cefazolin most frequent interval every $8 \mathrm{~h}$ \\
substitutions & (ie, every $6 \mathrm{~h}$ dispensed every $8 \mathrm{~h}$ ) \\
& Clindamycin most frequent interval every \\
& $8 \mathrm{~h}$ (ie, every $6 \mathrm{~h}$ dispensed every $8 \mathrm{~h}$ ) \\
& Metronidazole/cefazolin for cefotetan \\
& Oral metronidazol dispensed for all oral \\
vancomycin unless no substitute indicated \\
Restricted drugs & Intravenous ciprofloxacin \\
& Meropenem \\
\hline
\end{tabular}

TABLE 3

Antimicrobial drug use evaluations in the 1990s at the Victoria General and Royal Jubilee Hospitals in the Capital Health Region of British Columbia

\begin{tabular}{ll}
\hline Drug use evaluation & Year \\
\hline Cefoxitin & 1990 \\
Cefotetan & 1992 \\
Ceftriaxone & 1993 \\
Cefotaxime & 1995 \\
Imipenem & 1997 \\
Vancomycin & 1998 \\
\hline
\end{tabular}

ditions have been met. Often, if these details are not established before the policy is made, they make take several months to resolve. A revision will be required to make the policy feasible.

Control programs: Antimicrobial 'control programs' are often implemented by pharmacy and therapeutics committees, and restrict the prescription of antimicrobial drugs in some manner. A number of control strategies have been employed (Table 2). Control programs often provide quick and reliable cost savings, sometimes at the expense of individual patient needs. Although the process for mandating the programs includes peer review by several medical committees, such as the Antimicrobial Review, Pharmacy and Therapeutics, and Medical Advisory committees, the majority of prescribers only become aware of programs when their prescriptions are either refused or altered by the pharmacy department. For several years, a reserved antimicrobial drug (RAD) program was employed as an antimicrobial control program at the Victoria General and Royal Jubilee hospitals. The program involved the restriction of certain antimicrobials based on approved indications. Following the prescription of a reserved drug, the prescriber would be sent a RAD form to be filled out to ensure continued supplies of the medication from the pharmacy. A clinical pharmacist would review all forms for compliance with the approved indications. For years the program proved successful; however, it became more laborious than initially intended when some prescribers were readily filling out too many forms. Five prescribers were filling out 30 forms annually, rather than 50 prescribers filling out three forms annually. Over the past five 
TABLE 4

Contents of the antimicrobial handbook for the Capital Health Region of British Columbia

Antibiotic review subcommittee
Antimicrobial costs
Antibiogram tables/footnotes
Laboratory protocol notes
Bacterial endocarditis prophylaxis guidelines
Surgical antibiotic prophylaxis guidelines
Timing of prophylactic postoperative antibiotic administration
Guidelines for the empiric treatment of febrile neutropenia (adults)
Flow chart for the empiric treatment of febrile neutropenia (pediatrics)
Restricted antimicrobial drugs
Sequential antimicrobial therapy
Parenteral to oral antibiotic conversion regimens (adult)
Parenteral to oral antibiotic conversion regimens (pediatric)
Clostridium difficile associated colitis
Once daily aminoglycoside dosing
Vancomycin use recommendations
Infection control
$\quad$ Accidental exposure to blood/body fluids
Recommendations for hepatitis B prevention in health care
$\quad$ werkers
$\quad$ enterococci

years most of the drugs were removed from the RAD program, and recently it has been officially abandoned.

Drug usage evaluations: A number of antimicrobial drug usage evaluations have been performed over the past years (Table 3). The evaluations have largely been retrospective chart reviews of patient health records. The following are steps in the review process:

- drug evaluation endorsed by the Pharmacy and Therapeutics Committee;

- review process overseen by the Antimicrobial Subcommittee;

- appropriate indications for use set out by local panel of experts;

- review done by the Pharmacy Department in a timely manner; and

- formal review report and recommendations presented to the Pharmacy and Therapeutics Committee.

In the early 1990s, although drug usage evaluations were successful in decreasing drug use of the 'review drug', other drugs not in the review spotlight soon became overused.

Information/education: Providing antimicrobial prescribers with local practice information has proven invaluable. Most physicians are painfully unaware of antimicrobial costs or antibiogram data in their hospitals. Over the years several documents were produced that related to antimicrobial cost comparisons and antimicrobial use guidelines, including sur-

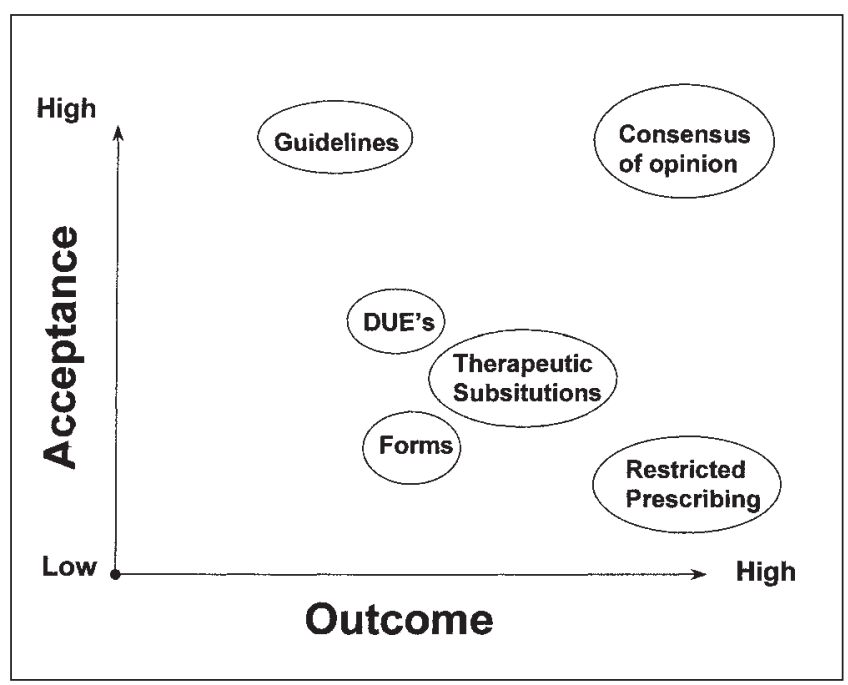

Figure 2) Strategies for optimizing antibiotic use; DUE Drug use evaluation.

gical prophylaxis, endocarditis prophylaxis, febrile neutropenia and intravenous to oral sequential therapy guidelines. The documents have recently been collated in a handbook that is distributed to the medical and nursing staff (Table 4).

Infection control: An important companion of the antimicrobial drug use program is the ongoing surveillance and work done by infection control. The follow-up and reporting of postsurgical infection rates and antimicrobial drug resistance (eg, methicillin-resistant Staphylococcus aureus and vancomycinresistant enterococci) is of paramount importance.

Reaching consensus: Perhaps the real lesson to be learned in any stewardship program is reaching consensus. Different strategies may provide seemingly similar positive outcomes; however, the adoption and ongoing success of the strategies is often dependent on their acceptance by medical staff. Restricting antimicrobial prescription works to decrease drug use but is rarely embraced by prescribers as a whole. Also, antimicrobial drug use guidelines are informative but are rarely followed or even located when they are needed.

Strategies were most successful when the prescribers involved were consulted for input into the decision-making. A consensus was invariably reached after prescribers were provided actual data of current practices, listened to and provided consultation. While these steps do not define a winning formula, they do comprise a process that provides the necessary background information to identify problems that must be overcome before a true change of practice can occur. For example, once the Antimicrobial Subcommittee and surgeons agreed on the prophylactic regimen for the surgical prophylaxis guidelines, the regimen was incorporated directly into preprinted physicians' orders for the surgery. Thus, individual surgeons no longer had to chose or remember the guidelines agreed upon. Preprinted orders are reviewed annually, and changes are made accordingly. The best scenario is the consensus of opinion reached by key stakeholders when presented with local data. Initially, intravenous cefuroxime was ordered as antimicrobial prophylaxis perioerative to open heart surgery. A change from intravenous cefuroxime to intra- 
venous cefazolin was made by the Antimicrobial Subcommittee following a discussion with the cardiac surgeons. Subsequently, the physician preprinted orders were changed, and postoperative infection rates were monitored. Because infection rates were unchanged, cefazolin has continued to be the surgical prophylactic agent for our patients.

\section{ANTIMICROBIAL RESISTANCE}

Resistance to antimicrobial therapy has become a critical incentive, perhaps even greater than drug cost, for promoting appropriate antimicrobial use. While the threat of resistance was previously predicted, most hospitals are now facing real problems involving antimicrobial resistance. Resistance patterns in the Victoria General and Royal Jubilee hospitals have so far been low, with methicillin-resistant $S$ aureus, intermediate penicillin-resistant pneumococci and vancomycinresistant enterococci accounting for $2 \%, 10 \%$ and less than $1 \%$ of all $S$ aureus, pneumococci and enterococci isolates, respectively; however, an increase in organisms with inducible beta-lactamase production is being encountered. Perhaps early implementation of antimicrobial use strategies in the 1980s explains the low antimicrobial resistance patterns.

\section{SUMMARY}

The initial impetus for antimicrobial rationing was increased drug costs. At present, hospitals are also faced with real problems of drug resistance. A number of strategies have been used to improve antimicrobial drug use; however, no one strategy will work for all organizations or situations. Reaching consensus is integral to the development of strategies that promote optimal antimicrobial use.

The following are lessons that were learned during the development of the antibiotic stewardship programs at the Victoria General and Royal Jubilee hospitals.

- Drug use evaluations should be disease focused, not drug focused.

- 'University-developed' programs should not be expected to work automatically in 'community' settings.

- Antibiotic use in hospitals will affect the community and vice versa.

- Meeting with key stakeholders and reaching consensus should be the first step, not the last. 


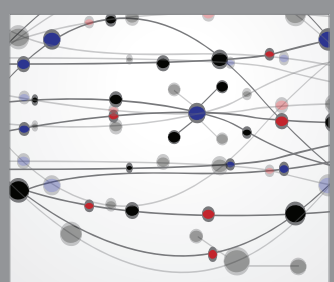

The Scientific World Journal
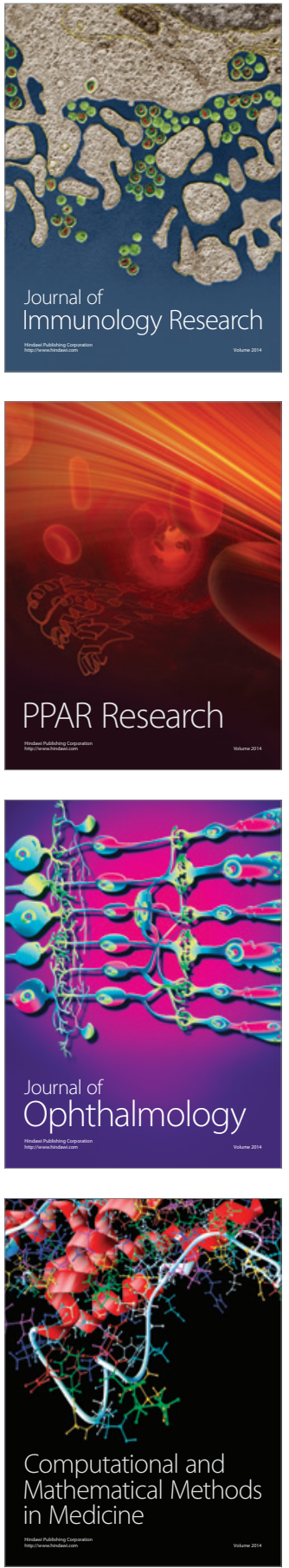

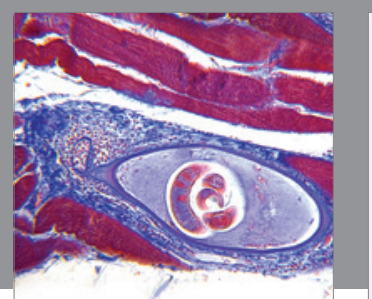

Gastroenterology Research and Practice

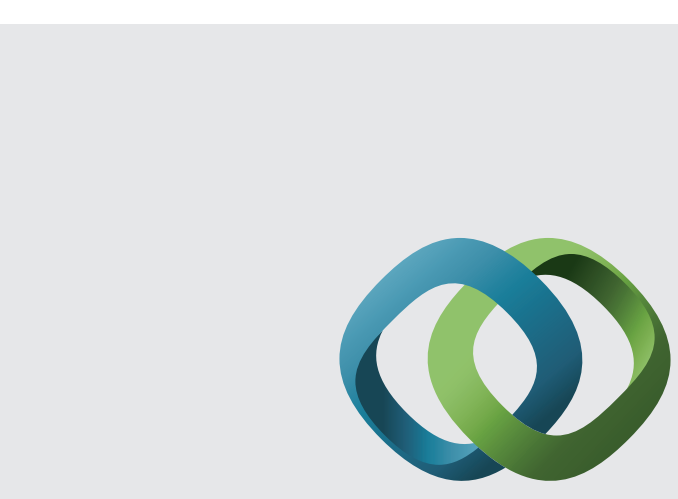

\section{Hindawi}

Submit your manuscripts at

http://www.hindawi.com
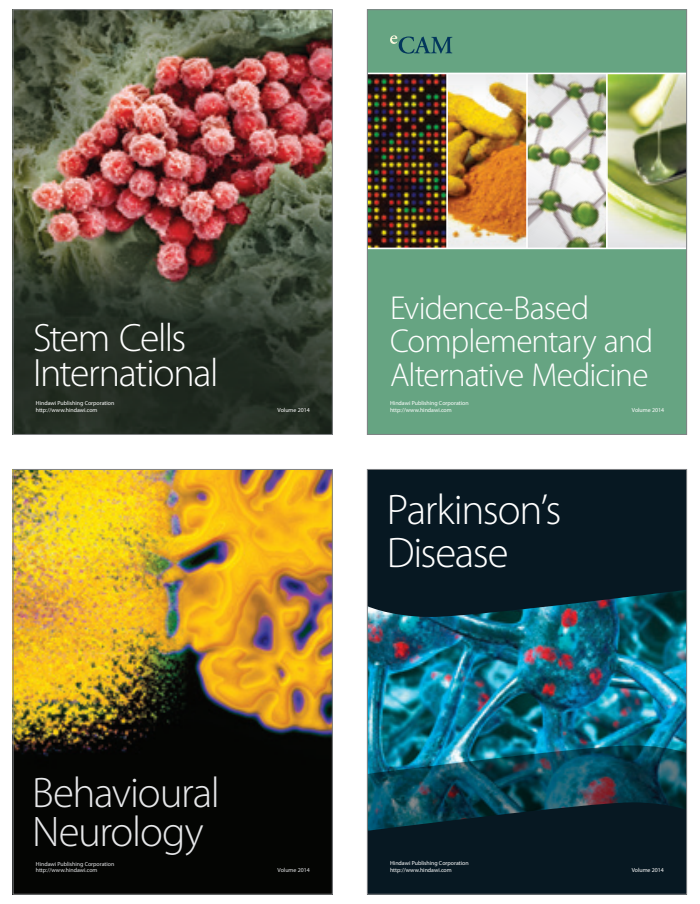
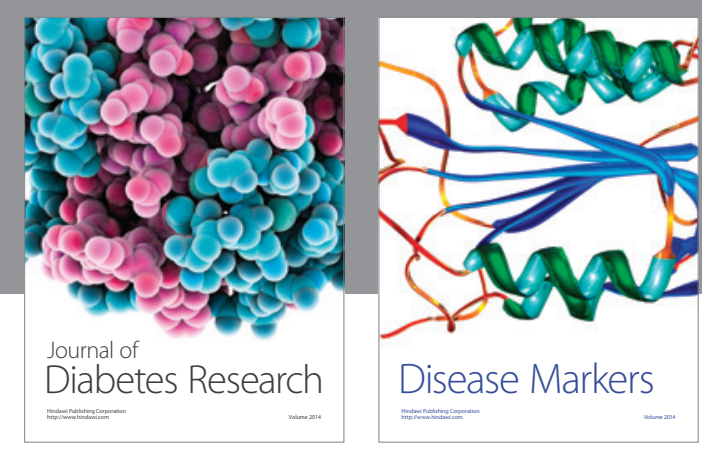

Disease Markers
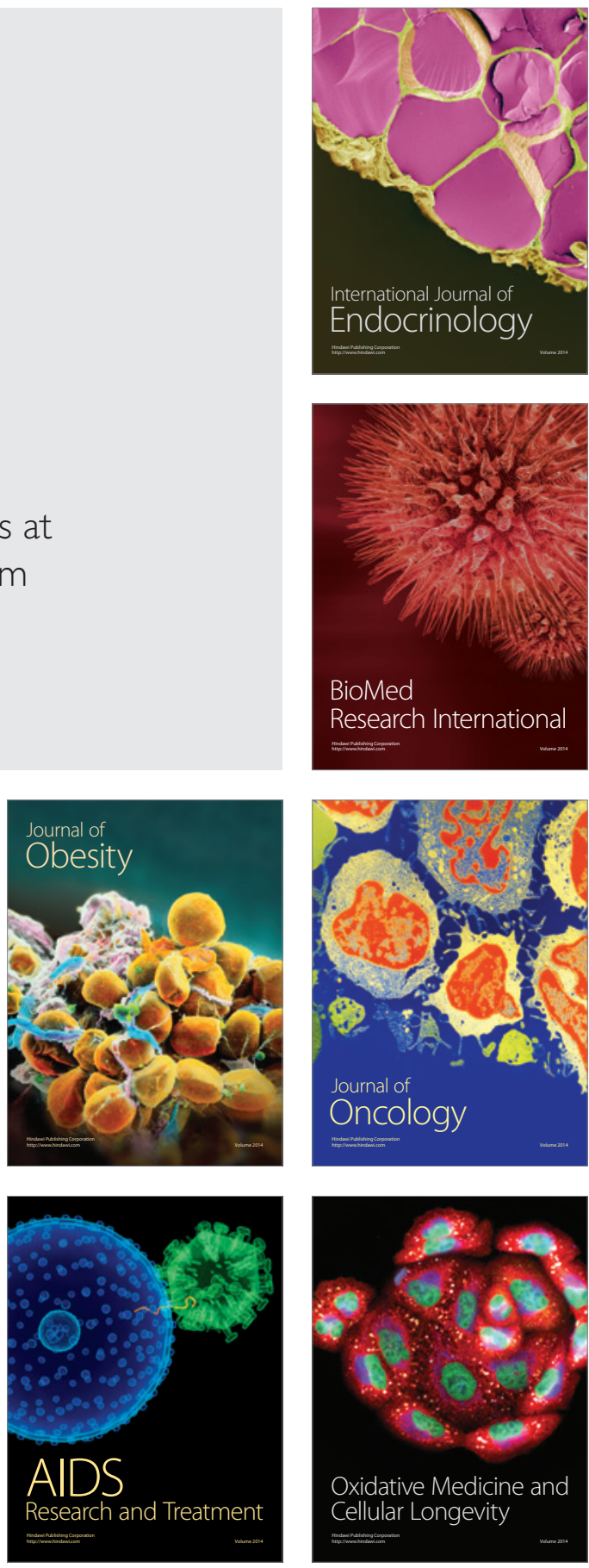